\title{
An Introduction to Regression Analysis on Parameters Selection

$$
\text { in Beltline Moulding Process }
$$

\author{
Abdul Talib Bon (Corresponding author) \\ Department Informatique - Laboratorie L3i, Pole Sciences et Technologie \\ Universite de La Rochelle \\ 17042 La Rochelle, Cedex 1, France \\ Tel: 60-12-766-5756_E-mail: talibon@gmail.com \\ Jean Marc Ogier \\ Department Informatique - Laboratorie L3i, Pole Sciences et Technologie \\ Universite de La Rochelle \\ 17042 La Rochelle, Cedex 1, France \\ Tel: 33-05-4645-8215 E-mail: jean-marc.ogier@univ-lr.fr \\ Ahmad Mahir Razali \\ School of Mathematical Sciences, Faculty of Science and Technology \\ Universiti Kebangsaan Malaysia \\ 86000 Bangi, Malaysia \\ Tel: 60-17-888-6805 E-mail: mahir@pkrisc.cc.ukm.my
}

\begin{abstract}
The world of manufacturing industries is forced to meet the demand of the end users in many different aspects especially to reduce the number of defects and production cost. Since then, the manufacturers have many introduced techniques and strategies in order to achieve zero defects for end products. Therefore, this research is an early attempt to introduce a proper method for manufacturers to achieve their goal starting from parameters selection and then optimization to control the belt line moulding production process. We apply regression analysis to make parameters selection and then used the best variables selected to optimize or in this case to minimize defects in belt line moulding process. The findings from this study we found from the correlation model only three parameters have strong correlation of fourteen parameters were studied. The results are very useful evidence and applicability to beltline moulding manufacturer for implementation.
\end{abstract}

Keywords: Belt line moulding, Regression analysis, Parameters selection, Process

\section{Introduction}

The manufacturing industries in Malaysia nowadays go toward in the competition scenario from light industries to heavy industries. The competitions not only based on quantity of sales or products but they are looking on how the product precise can be meet from industry to satisfy what the end user needs. Therefore, all manufacturing industries must be capable to produce services or product took initiatives to customer's needs and desire at the best quality product and minimum cost. In this research the authors study more specific area in beltline moulding in automotive manufacturing. Beltline moulding is a process with many variations in raw materials, machinery conditions and ambient conditions. It also has a temporal aspect where line conditions change during operation, affecting the end product. Typical process control procedures include statistical analysis of periodic batch samples, control charts of sample mean or range, and trial and error. Beltline moulding companies are concerned with automating information flow and the use of intelligent computer controls can play an important role 


\section{Significance and Benefits of Proposed Research}

The application of quantitative technique in improving a product process thus far is still a recent phenomenon. There is an urgent need for more objectives, realistic and accurate model for future planning and policy evaluation. This is quite obvious as the automotive manufacturing sector (beltline part of car body) undergoes structural changes and is becoming more complex due to technological advances, manufacturing management, product demand and competition from other manufacturer.

\section{The Objective of the Study}

In view of the importance of having such tools, the study aims to achieve the following objectives:

i. To select the best parameter settings from the four factors.

ii. To apply Correlation Modelling approach for parameter selection.

\section{The Scope of the Research}

The key issue that to some extent would limit the reliability and validity of the analysis is the availability of data that are relevant to scope of the study. Moulding manufacturing is known to be affected by many factors like material, machine, measurement, human etc. The detail list of the dependent and independent variables used in this research are suggested by many researchers in manufacturing and one of the known as Yazici (1990). The data used in this study are cited from daily data from beltline moulding manufacturer for Malaysia's national car. The area of this study will be determined later depend on the availability of the data set. At this stage, the authors are preparing memorandum of understanding $(\mathrm{MoU})$ in research collaboration between moulding Manufacturer Company and the authors.

\section{Literature Review}

\subsection{Belt Line Moulding Process}

\subsubsection{Extrusion Process}

The very important part in roll forming process is extrusion process. Basically many definitions authors found about extrusion which is ampef.com defined extrusion is process by which polymer is propelled continuously along a screw through regions of high temperature and pressure where it is melted and compacted, and finally forced through a die (slit) to form a thin film.

Meanwhile, Ampef. (2002) defined extrusion as a forming technique whereby a material is forced, by compression, through a die orifice, and Jason, Leadbitter, (2004) defined it is a method of processing plastics where the material is pushed through a die under pressure to form a continuous strip of a particular shape. Additionally, extrusion is a fabrication process in which a heat-softened polymer is forced continually by a screw through a die Seymour and Carraher, (1993). The extrusion can be further defined as the process of manufacturing and/or shaping a material by forcing it through a die (RoofHelp.com, 2006).

\subsubsection{Roll Forming Process}

In the manufacture of automobile mouldings, especially the belt line mouldings that border the interface between a car door panel and the bottom outside edge of the door windows, it has become aesthetically fashionable to provide a strip of stiff decorative or ornamental plastic material on the outer or inner side of the arch or channel shaped moulding in combination with the coil look of an exposed portion of the core material. In addition to these aesthetic functions, the inner portion of the moulding comprises a flocked elastomeric lip adapted to bear against the window, sealing the door from the elements, and providing a guide for reciprocating movement of the window.

\section{Research Methodology}

The research purpose of modelling in manufacturing industry is to apply parameters selection analysis using regression and correlation analysis. Regression analysis is used to reduce a set of large number of variables to a smaller number of variables. The regression model used is as shown below:

$$
Y=\beta_{0}+\beta_{1} X_{1}+\beta_{2} X_{2}+\ldots+\beta_{m} X_{m}+\varepsilon
$$

where $Y=$ Response variable (dependent)

$$
\begin{aligned}
& \beta_{0} \ldots \beta_{m}=\text { Parameters } \\
& X_{1} \ldots . X_{m}=\text { Predictor variables (independent) }
\end{aligned}
$$

and $\varepsilon$ is a random error term.

The technique of least squares is used to estimate the parameters $\beta_{0}, \beta_{l}, \ldots \ldots, \beta_{m}$. In this technique, we minimizing the sum of the squared differences of the actual $Y$ values and the values of $Y$ predicted by the regression equation. The computation can be shown easily in matrix notation. Suppose $\boldsymbol{Y}$ is a vector of observed values of $\boldsymbol{Y}$, the $\boldsymbol{X}$ matrix is a matrix for the independent variables, and the $\varepsilon$ as an error vector such that; 


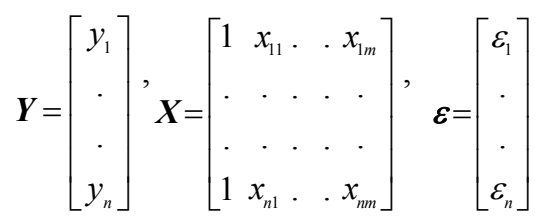

Then the regression model can be represented in the matrix notation as

$$
\boldsymbol{Y}=\boldsymbol{X} \boldsymbol{\beta}+\boldsymbol{\varepsilon}
$$

Where is $\beta^{\prime}=\left(\beta_{0}, \beta_{1}, \ldots, \beta_{\mathrm{m}}\right)$ is the vector of parameters. By solving the set of normal equations given by

$$
X^{\prime} X \boldsymbol{\beta}=\boldsymbol{X}^{\prime} \boldsymbol{Y}
$$

and assuming the matrix $\boldsymbol{X}^{\prime} \boldsymbol{X}$ is non-singular, a unique solution will be found which is given by

$$
\hat{\boldsymbol{\beta}}=\left(\boldsymbol{X}^{\prime} \boldsymbol{X}\right)^{-1} \boldsymbol{X}^{\prime} \boldsymbol{Y}
$$

Correlation analysis is a technique for investigating the relationship between two quantitative or continuous variables. The correlation coefficient, $r$ is a measure of the strength of the association between the two sets of variables. The most common correlation coefficient is the Pearson's correlation coefficient which is defined as

$$
r=\frac{\sum_{i=1}^{n}\left(x_{i}-\bar{x}\right)\left(y_{i}-\bar{y}\right)}{\sqrt{\left[\sum_{i=1}^{n}\left(x_{i}-\bar{x}\right)^{2}\right]\left[\sum_{i=1}^{n}\left(y_{i}-\bar{y}\right)^{2}\right]}}
$$

where $\left(x_{i}, y_{i}\right)$ are pairs of sample points $\bar{x}$ and $\bar{y}$ are the means of measurements on the variables. Generally, the correlation coefficient varies from -1 to +1 .

\section{Results and Discussions}

In this research, we shall discuss the analysis of the relationship between two quantitative outcomes using scatter plot. A scatter plot is simply a cloud of points of the two variables under investigation. From the scatter plot, we can see very clearly whether there is a linear association between the two variables and guess accurately the value of the correlation coefficient. After looking a scatter plot, we then go ahead and confirm the association by conducting a correlation analysis. We use the correlation coefficient, $r$ to describe the degree of linear relationship between the two variables.

Table 1 gives a guideline on the strength of the linear relationship corresponding to the correlation coefficient value. Values near 0 means no (linear) correlation values near \pm 1 means very strong correlation. The negative sign means that the two variables are inversely related, that is, as one variable increases the other variable decreases.

The scatter plots of sets of data with varying degrees of linear association, we found the closer to zero the correlation coefficient is, the less the points fall on a straight line (hence the term "linear" correlation coefficient). For the cylinder factor, where are have six parameters been involved in this analysis. Where are cylinder 1 (CY1), cylinder 2 (CY2), cylinder 3 (CY3), cylinder 4 (CY4), adapter (ADPT) and die. From the correlation analysis we found CY1 and CY2 have strong correlation coefficients with 0.927 and between CY2 and CY3 with 0.839 . While, strong correlation also between CY1 and CY4 with Pearson's $r=0.873$. All of the very strong correlations in this factor fall the positive correlation. From the correlation we can summary result base on very strong correlations. The parameters involved are CY1, CY2 and CY4 as shown as Table 2. From the Table 2, we can illustrate to 3-Dimension graphic as Figure 1 shown very strong relationship between CY1, CY2 and CY4 in the cylinder factor.

Factors score covariance matrix shown as Table 3 that although theoretically the factor scores should be entirely uncorrelated the covariance is not zero, which is a consequence of the scores being estimated rather than calculated exactly.

The scatter plot for heater factor, we found not have any strong correlation between parameters where heater no. 1 (current unit), H1_C; heater no. 1 (temperature unit), H1_T; heater no. 2 (current unit), H1_C; and heater no. 2 (temperature unit), H1_T. That means no any correlation between four parameters. The plot indicates a lack of a relationship between the all variables with Pearson correlation coefficient between -0.015 to 0.332 with $\mathrm{p}$-value is 0.05 levels (2-tailed).

The scatter plot for power panel with four parameters are looper, roller, pulling and cutter, most of the correlation coefficient is to negative one, the more the points will fall along a line stretching from the upper left to the lower right. However, looper and roller have strong correlation with 0.790 , with a 2 -sided $1 \%$. Table 4 from the output listing shown gives the actual value of the correlation coefficient along with its p-value. The correlation coefficient is 0.790 and the p-value is 0.01 . From these values, it can be concluded that the correlation coefficient is significant beyond the 1 percent level. In other words, looper had moderately strong correlation with roller. From the coil factor all the parameters which is coil thickness, width and burr where no any correlation to each others 
have. That shown very weak correlation in this factor. A relationship between all parameters is not apparent from the plot, Pearson correlation coefficient less than $0.3(\mathrm{p}<0.05)$.

Besides that the scatter plot for raw material composition factor shows some degree of association between tensile strength and elongation break which the Pearson correlation coefficient, $r$ is about $+0.789(p<0.01)$. The Table 5 from the output listing are shown below. Notice that the Pearson correlation coefficients are exactly 0.789 and significant beyond the 1 percent level. The association between the two parameters is significant indicating that the two parameters the 98 data in a similar way.

The closer the correlation coefficient is to one, the more the points will fall along a line stretching from the lower left to the upper right. The scatter plot for ambient conditions factor clearly shows a linear association between the two variables air velocity and air exchange rate coefficient of correlation which $r$ is +1 . Data lie on a perfect straight line with a positive slope. This indicates as the air velocity score get higher, so will the air exchange rate in higher. Meanwhile, the others correlation between air velocity with temperature and relative humidity, temperature with relative humidity, and relative humidity with air exchange rate do not show any degree of linear association at all, $r$ less than 0.4 .

\section{Conclusion}

We can conclude from the Correlation modelling analysis for six factors not all factors gave the strong correlation between parameters. We found that model for selected parameters involved in beltline moulding process factor as shown in Table 6 as a conclusion. We select correlation coefficient value at least 0.8 which shown very strong in strength of linear relatively.

\section{References}

Ampef. (2002). When the world thinks of plastic films, it thinks of PET: Glossary Terms. [Online] Available: http://www.ampef.com/gloss.html

H. Yazici. (1990). Implementation of SPC techniques in the PVC pipe industry. Engineering Management Journal, 2(3), 59-64.

Jason, Leadbitter, (2004), NZ Newsletter Issue 13. [Online] Available: http://www.hydropolymers.com/en/ media_room/glossary (January 2004)

M. Pagano \& K Gauvreau. (2000). Principles of Biostatistics. (2nd ed.). Duxbury: Pacific Grove, CA.

RoofHelp.com, (2006), What's Inside: Glossary [Online] Available: http://www.roofhelp.com/Glossary/glossary_e.htm R. J. Freund \& R. C. Little. (2000). SAS ${ }^{\circledR}$ System for Regression. (3rd ed.). Cary, NC: SAS Institute Inc.

Seymour \& Carraher, (1993), Polymer Chemistry Dekker [Online] Available: http://www.matse1. mse.uiuc.edu/ tw/ polymers/glos.html

Y. H. Chan. (2003). Biostatistics 104: Correlational Analysis. Singapore Medical Journal, 44(12), 614-619. 
Table 1. Strength of Linear Relationship

\begin{tabular}{cc}
\hline Correlation Coefficient value & Strength of linear relationship \\
\hline At least 0.8 & Very strong \\
0.6 up to 0.8 & Moderately strong \\
0.3 to 0.5 & Fair \\
Less than 0.3 & Poor \\
\hline
\end{tabular}

Source: Y H Chan (2003)

Table 2. Correlation Coefficients between CY1, CY2 and CY4

\begin{tabular}{|l|l|l|}
\cline { 2 - 3 } \multicolumn{1}{c|}{} & CY2 & CY4 \\
\hline CY1 & 0.927 & 0.873 \\
\hline CY2 & 1 & 0.839 \\
\hline
\end{tabular}

Table 3. Factor Score Covariance Matrix

\begin{tabular}{ccc}
\hline Factor & CY2 & CY4 \\
\hline CY2 & 17.246 & 9.817 \\
CY4 & 9.817 & 7.938 \\
\hline
\end{tabular}

Table 4. Correlation between Looper and Roller Correlations

\begin{tabular}{|lc|l|l|}
\cline { 3 - 4 } \multicolumn{1}{c|}{} & \multicolumn{1}{c|}{ Looper } & \multicolumn{1}{c|}{ Roller } \\
\hline \multirow{2}{*}{ Looper } & Pearson Correlation & 1.000 & $.790^{* *}$ \\
& Sig. (2-tailed) &. & .000 \\
$\mathrm{~N}$ & 43 & 43 \\
\hline \multirow{2}{*}{ Roller } & Pearson Correlation & $.790^{* *}$ & 1.000 \\
& Sig. (2-tailed) & .000 &. \\
& $\mathrm{~N}$ & 43 & 43 \\
\hline
\end{tabular}

** Correlation is significant at the 0.01 level (2-tailed)

Table 5. Correlation between Tensile Strength and Elongation Break Correlations

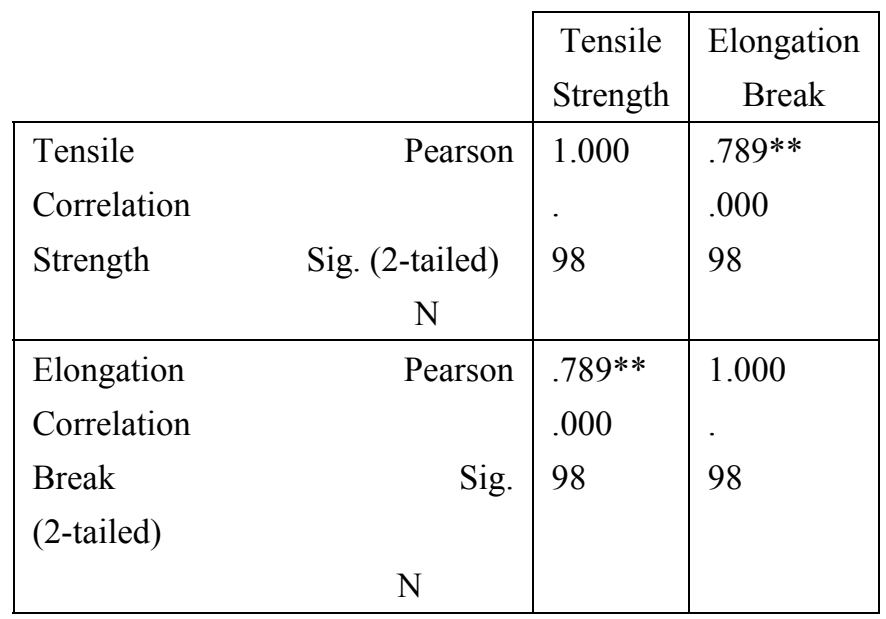

** Correlation is significant at the 0.01 level (2-tailed) 
Table 6. Model for Strong Correlation for Selected Parameters

\begin{tabular}{|l|l|l|l|}
\cline { 2 - 4 } \multicolumn{1}{c|}{} & CY1 & CY4 & Air velocity \\
\hline CY2 & 0.93 & 0.839 & - \\
\hline CY4 & 0.87 & 1.0 & - \\
\hline Air Exchange Rate & - & - & 1.0 \\
\hline
\end{tabular}

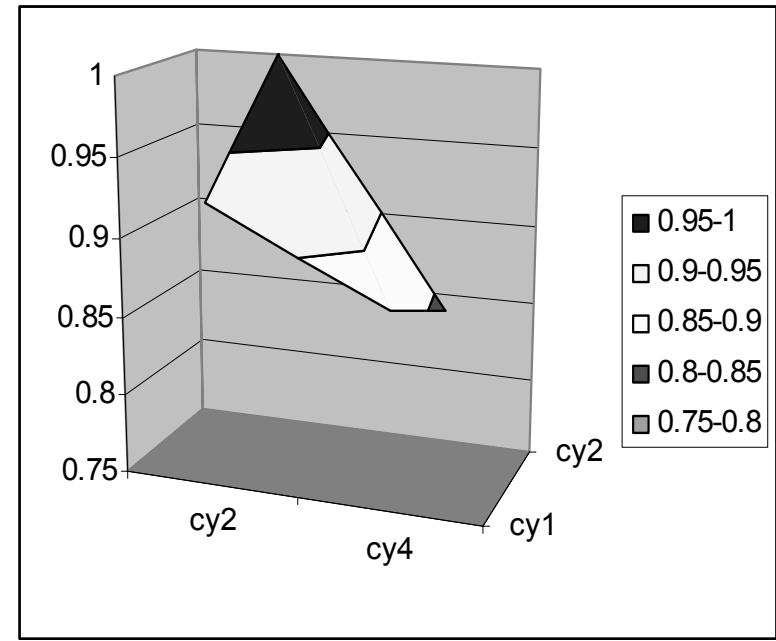

Figure 1. Correlation Graph between CY1, CY2 and CY4 